\title{
Erratum
}

Oil \& Gas Science and Technology - Rev. IFP, Vol. 62 (2007), No. 3, p. 415

Copyright (C) 2007, Institut français du pétrole

DOI: $10.2516 /$ ogst:2007035

\section{Geomechanical Log Deduced from Porosity and Mineralogical Content}

Diagraphie géomécanique déduite de la porosité et de la composition minéralogique

\section{E. Bemer, O. Vincké and P. Longuemare}

\author{
Institut français du pétrole, IFP, 1 et 4, avenue de Bois-Préau, 92852 Rueil-Malmaison Cedex - France \\ e-mail: elisabeth.bemer@ifp.fr - olivier.vincke@ifp.fr - pascal.longuemare@ifp.fr
}

Oil \& Gas Science and Technology - Rev. IFP, Vol. 59 (2004), No. 4, pp. 405-426

Copyright (C) 2004, Institut français du pétrole

DOI: $10.2516 /$ ogst:2004028

A misprint occurred in the expression of the parameters of equations 6 and 7. Parameters $A_{n}$ and $B_{n}$ (p. 408) should read:

$$
\begin{gathered}
A_{n}=\sum_{i=1}^{n-1} \frac{f_{i}}{\left(K_{i}-K_{n}\right)^{-1}-\alpha_{n}} \\
B_{n}=\sum_{i=1}^{n-1} \frac{f_{i}}{\left[2\left(G_{i}-G_{n}\right)\right]^{-1}-\beta_{n}}
\end{gathered}
$$

Les expressions des paramètres $A_{n}$ et $B_{n}$, aux équations 6 et 7 (p. 408), comportent une erreur. Leur forme correcte est la suivante :

$$
\begin{gathered}
A_{n}=\sum_{i=1}^{n-1} \frac{f_{i}}{\left(K_{i}-K_{n}\right)^{-1}-\alpha_{n}} \\
B_{n}=\sum_{i=1}^{n-1} \frac{f_{i}}{\left[2\left(G_{i}-G_{n}\right)\right]^{-1}-\beta_{n}}
\end{gathered}
$$

